\title{
Nocturnal hypoxia and arrhythmias in patients with impaired left ventricular function
}

\author{
T Cripps, G Rocker, J Stradling
}

\begin{abstract}
Objectives-To document the incidence of hypoxic episodes in a series of patients with impaired left ventricular function, and to correlate the occurrence of hypoxia with severity of arrhythmia.

Patients-34 patients with breathlessness and clinical evidence of left ventricular dysfunction.

Main outcome measures-Simultaneous overnight finger oximetry and electrocardiographic monitoring.

Results-High grade arrhythmias (Lown grade > III) occurred in 20/34 $(59 \%)$ of patients, and frequent dips in oxygen saturation were noticed (mean dip frequency $4 \cdot 8 / \mathrm{h}$, range $0 \cdot 1-20 \cdot 0) .20 / 34$ $(59 \%)$ of patients had episodic hypoxaemia, including $13 / 34(38 \%)$ with a classical Cheyne Stokes pattern. There was a correlation between dip frequency and the presence of high grade arrhythmias (those with high grade arrhythmia had mean (SD) $6.7(5.5)$ dips/h $v 2.2(3.4)$ in those without, $p<0.01$ ); there was also a correlation between the presence of arrhythmias and episodic hypoxaemia (episodic hypoxaemia in those with high grade arrhythmias occurred in $17 / 20$ $(85 \%) v 3 / 14(21 \%)$ of those without arrhythmias, $p<0.002)$. There was no correlation between the presence of high grade arrhythmias or dip frequency and the extent of left ventricular impairment, which was present in all patients (mean (SD) ejection fraction $26 \%$ $(13 \%))$.
\end{abstract}

Conclusion-Noticeable abnormalities of nocturnal oxygen saturation occur in patients with impaired left ventricular function, and these are associated with high grade arrhythmias. Interventions that limit desaturation may have valuable anti-arrhythmic effects.

Department of Radcliffe Hohn Headington, Oxford and Osler Chest Unit, Churchill Hospital, Oxford

T Cripps

G Rocker

J Stradling

Correspondence to Dr Dr T Cripps, Cardiac Department, John Radcliffe Hospital, Headington, Oxford OX39DU.

Accepted for publication 12 March 1992 greatest expression of the arrhythmoge effect of hypoxia might, however, be expected in those with intrinsic myocardial disease, particularly in patients with impaired left ventricular function, in whom ventricular arrhythmias are frequent and easily precipitated..$^{56}$ The purpose of this study was to examine fluctuations in nocturnal oxygen saturation in a group of patients with impaired left ventricular function, and to correlate these fluctuations with the occurrence of ventricular arrhythmias and the severity of left ventricular impairment.

\section{Patients and methods} PATIENTS

Patients were selected from those admitted to hospital for elective cardiac catheterisation, or for treatment of refractory chronic heart failure. Those considered to have impaired left ventricular function were identified clinically on the basis of a history of dyspnoea, a cardiothoracic ratio $>50 \%$ on a chest radiograph, and clinical signs suggestive of left ventricular impairment. All had New York Heart Association (NYHA) grade II-IV dyspnoea. Patients were excluded if they gave a history suggestive of chronic bronchitis, asthma, chronic airways obstruction, or other intrinsic pulmonary disease, but formal lung function tests were not performed. None of the patients had symptoms suggestive of obstructive sleep apnoea, and none were grossly obese ( $>115 \%$ of ideal body weight). Each patient gave informed consent; the study was approved by the hospital ethics committee.

\section{OXIMETRY}

Overnight oximetry was performed at the bedside with an Ohmeda Biox 3700 pulse oximeter (version $\mathrm{J}$ ) and a flexible finger probe. ${ }^{7}$ The device's internal memory stores a two digit value at the end of each 12 seconds, which represents the lowest oxygen saturation during those 12 seconds. Thus dips in saturation are not missed but recovery between dips may be underestimated. The 2400 values for oxygen saturation generated over an eight hour period were subsequently downloaded to an IBM compatible personal computer and analysed for mean saturation and dips in excess of $4 \%$. Dips were counted when the saturation fell more than $4 \%$ below the previous updated high. No further dip was recorded unless the saturation rose by more than $3 \%$. The probe was applied to the forefinger of either hand late in the evening as the patient was preparing to retire and was removed the next morning. We performed the study on the second or later night in hospital, so that the patient was accustomed to his surroundings, but formal 
conditioning was not carried out, and sleep stage and chest wall movements were not assessed. In no case was oxygen treatment required during the recording period.

\section{ELECTROCARDIOGRAPHIC MONITORING}

Conventional long-term electrocardiographic tape recording techniques were performed with an Oxford Medilog 4000-II recording and analysis system. Recordings were analysed for frequency of extrasystoles and modified Lown grade. Frequency of extrasystoles was divided into three bands: $\leqslant 10,>10 \leqslant 30$, and $>30$ extrasystoles in any hour. The Lown grade was dichotomised between those with Lown grade III or more (multiform, couplets, or nonsustained ventricular tachycardia) and those with Lown grade I or II (uniform and nonrepetitive forms). In all cases, the electrocardiographic recording period and the oximetry recording period were the same.

\section{MEASUREMENT OF EJECTION FRACTION}

Ejection fraction was estimated by conventional techniques, with either cineventriculography (Dodge RAO method) in patients undergoing cardiac catheterisation $(\mathrm{n}=21)$, and gated blood pool scanning $(n=10)$, or cross sectional echocardiography $(n=3)$ in those not undergoing catheterisation.

\section{CLINICAL ASSESSMENT}

All patients underwent a full clinical assessment, including a full history and physical examination, 12 lead electrocardiography and chest radiography. Drug treatment was continued unchanged; the treatment at the time of study was recorded. No patients were taking anti-arrhythmic agents.

\section{STATISTICS}

Continuous variables are expressed as mean (SD) and were compared by two tailed $t$ test. Discrete variables were compared by the $\chi^{2}$ test with Yates's continuity correction.

\section{Results}

PATIENTS STUDIED

A total of 37 patients were studied; in three cases, data were incomplete, leaving a study group with complete data comprising 34 patients. Of the incomplete cases, in two the Holter recording was destroyed due to a fault with the tape transport mechanism; one patient was excluded because of an inadequate oximetry recording. Good oximetry recordings proved feasible in patients with poor left ventricular function. The mean age of the study group was 60.5 (median 62 , range $27-80$ ) years and $2 / 34(6 \%)$ were women. In most $(29 / 34$, $85 \%$ ) the aetiology of the left ventricular disease was ischaemic; three $(9 \%)$ had dilated cardiomyopathy, one had valvar heart disease, and one had both valve and coronary disease. All were in NYHA classification grades II-IV for dyspnoea; nine grade IV, 12 grade III and 13 grade II. All were receiving treatment with diuretics and most were also receiving treat- ment with an angiotensin converting enzyme inhibitor. No patients were taking antiarrhythmic agents.

\section{OXIMETRY}

The mean (SD) saturation during the recording period was 93 (3)\%, median $94 \%$, range $84-97 \%$; four patients (12\%) had a mean saturation below $90 \%$.

Oximetry tracings were classified into three types: a recording without episodic hypoxia, when there was little fluctuation in oxygen saturation, with no dips below $90 \%$; sinusoidal fluctuations in oxygen saturation confirmed visually to correspond to Cheyne-Stokes respiration, with dips below $90 \%$ saturation; and other patterns where dips in oxygen saturation below $90 \%$ occurred (fig 1 ). A total of $14 / 34$ $(41 \%)$ showed no abnormality of oxygen saturation profile; $13 / 34(38 \%)$ showed the Cheyne-Stokes pattern, and $7 / 34(21 \%)$ had other patterns. The dip frequency ranged from $0.1 \mathrm{dips} / \mathrm{h}$ to $20 / \mathrm{h} ; 6 / 34(18 \%)$ had a dip frequency of $>10 / \mathrm{h}$. The mean dip frequency was $4 \cdot 8$ (median $2 \cdot 7$ ) dips/h. Dip frequency was $7 \cdot 3(6.6) \mathrm{dips} / \mathrm{h}$ in those with NHYA class IV dyspnoea, compared with $5 \cdot 1(5 \cdot 8) \mathrm{dips} / \mathrm{h}$ in those with class III dyspnoea (NS).

\section{ELECTROCARDIOGRAPHIC MONITORING}

A total of $20 / 34(59 \%)$ of patients had a frequency of extrasystoles of $\leqslant 10 / \mathrm{h} ; 10 / 34$ $(29 \%)>10$ and $\leqslant 30 / h$; four had $>30 / h$. Lown grade IVB arrhythmias (non-sustained ventricular tachycardia) were seen in 11/34 $(32 \%)$; IVA arrhythmias (couplets) were seen in $8 / 34(23 \%)$ and Lown grade <III arrhythmias in $14 / 34(41 \%)$. One patient had grade III arrhythmias (multifocal extrasystoles alone).

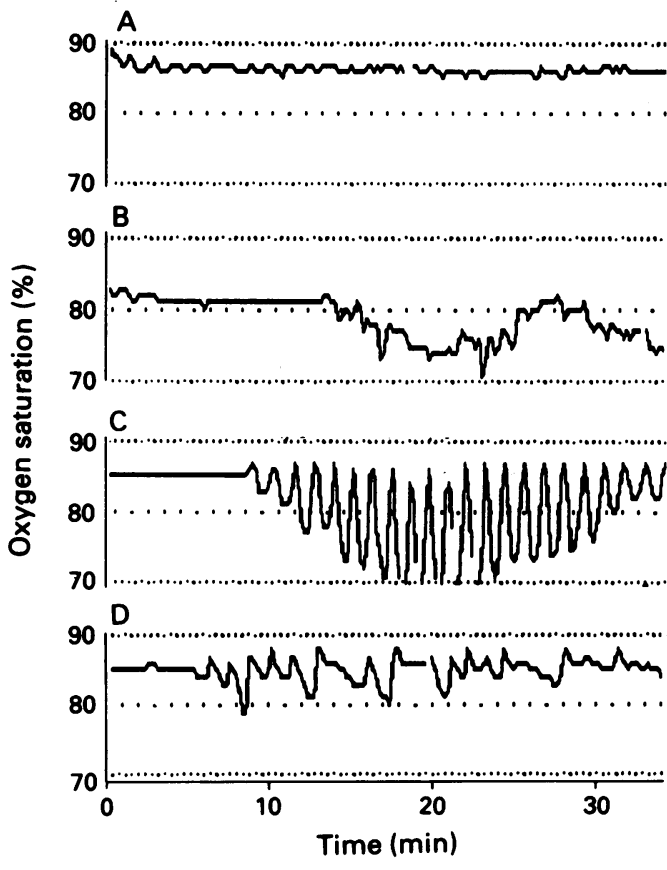

Figure 1 Patterns of oxygen saturation dips. (A) little fluctuation in saturation level, no dips below $90 \%$; (C) sinusoidal pattern due to Cheyne-Stokes respiration. (C) sinusoidal pattern 
Relation between arrhythmias and results of oximetry

\begin{tabular}{llll}
\hline & Lown $>$ III & Lown $<I I I$ & p Value \\
\hline Ejection fraction (mean (SD)\%) & $25(12)$ & $27(14)$ & NS \\
Dip frequency (mean (SD)/h & $6 \cdot 7(5.5)$ & $2 \cdot 2(3.4)$ & $<0.01$ \\
Mean (SD) saturation (\%) & $92.3(3.2)$ & $95(1.8)$ & $<0.02$ \\
Episodic hypoxia present & $17 / 20(85 \%)$ & $3 / 14(21 \%)$ & $<0.002$ \\
\hline
\end{tabular}

Dip frequency was significantly higher in those with frequent extrasystoles $(>10 / \mathrm{h})$ compared with those with infrequent extrasystoles (dip frequency $7 \cdot 1(6.0)$ in those with $>10$ compared with $3.0(4.0) \mathrm{dips} / \mathrm{h}$ in those with $\leqslant 10$ extrasystoles, $\mathrm{p}<0.02)$.

\section{EJECTION FRACTION}

In most cases, objective assessment showed poor left ventricular systolic function, with $28 /$ $34(82 \%)$ having an ejection fraction of $<45 \%$ and $16 / 34(47 \%)$ with an ejection fraction of $<30 \%$. The mean (SD) ejection fraction was $26(13) \%$ (median $25 \%$, range $10 \%-56 \%$ ). In three cases, ejection fraction was $>50 \%$, although all had been admitted to hospital with dyspnoea requiring diuretic treatment, had an increased cardiothoracic ratio on a chest radiograph, and had evidence of abnormalities of left ventricular wall motion. None of these three patients had hypoxic episodes. There was no difference between the mean ejection fraction in those with grade $>$ III arrhythmias compared with those with grade <III arrhythmias (25 (12)\% compared with 27 (14)\%).

\section{CORRELATION BETWEEN ARRHYTHMIAS AND OXIMETRY RESULTS}

The table shows the relation between severity of arrhythmia (Lown grade > III $v$ Lown grades I or II) and dip frequency, mean saturation, and ejection fraction. Saturation dips were more frequent in those with the more severe arrhythmias, whereas the ejection fraction was not significantly different between those with severe and those with less severe arrhythmias. Figure 2 illustrates the poor correlation between ejection fraction and dip frequency, showing that saturation dips are not merely a direct indication of severity of left ventricular dysfunction. A dissociation between clinical severity of disease and oximetry profile was also shown by the fact that

\footnotetext{
Figure 2 Scatter plot of ejection frequency
(LVEF) and dip frequency. There is no correlation between the two variables.
}

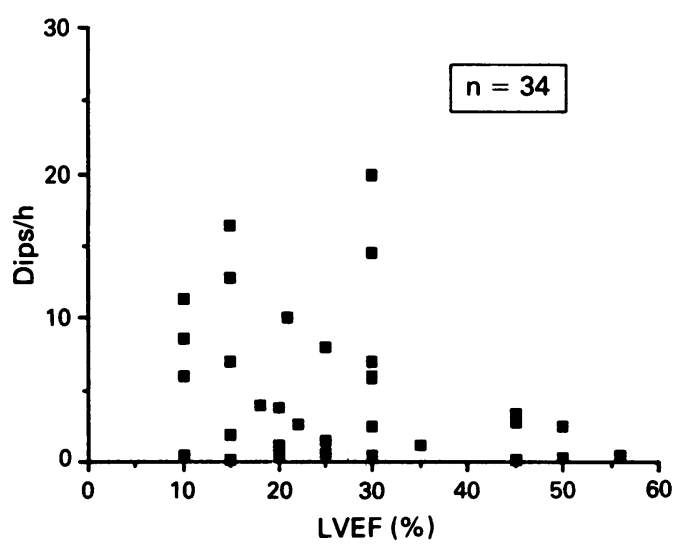

the mean dip frequency was no different between the clinically most severe cases (those with class IV NYHA dyspnoea) and those with class III dyspnoea.

The occurrence of episodic hypoxia was, however, correlated with severity of arrhythmia. The table shows Lown grade > III arrhythmias were only seen in $3 / 14(21 \%)$ cases where episodic hypoxia was absent but high grade arrhythmias were present in $17 / 20(85 \%)$ of those with episodic hypoxia.

Figure 3 shows that there was an inconsistent temporal correlation between episodic hypoxia and arrhythmias. In some cases, arrhythmias occurred during or just after hypoxic episodes, whereas in others, considerable episodic hypoxia did not result in arrhythmias. In other cases, arrhythmias occurred without hypoxia.

\section{Discussion}

Cardiac arrhythmias have been described during hypoxic episodes in patients with chronic airways obstruction and sleep apnoea, ${ }^{12}$ but few studies have investigated the relation between nocturnal hypoxia and arrhythmias in those with intrinsic myocardial disease. Otsuka et al correlated arrhythmias with oximetry in 81 patients with various cardiac disorders, but patients with left ventricular impairment were not specifically included. ${ }^{8}$ Dark et al showed an improvement in oxygen saturation after treatment of heart failure in six patients with left ventricular dysfunction, but no analysis of arrhythmia was undertaken. ${ }^{9}$ Hanly et al carried out oximetry in 10 outpatients with chronic heart failure and showed poor quality of sleep with frequent dips and arousals, but again arrhythmias were not analysed. ${ }^{10}$ Takasaki et al showed that continuous positive airway pressure treatment improved saturation and left ventricular function in patients with heart failure but again information concerning arrhythmias was not provided. ${ }^{11}$ Recently, Davies et al published a study of oximetry in 10 patients with heart failure: only one had non-sustained ventricular tachycardia, although this arrhythmia did appear to be temporally associated with a hypoxic episode. ${ }^{12}$

We have carried out arrhythmia and oximetry analysis in 34 patients with impaired left ventricular function. High grade arrhythmias (Lown grade III and above) were common, occurring in 59\%. Oximetry showed abnormal patterns in $50 \%$ of cases, the mean dip frequency being $4.8 \mathrm{dips} / \mathrm{h}$. A strong correlation was found between the occurrence of high grade arrhythmias and the presence of saturation abnormalities. A total of $17 / 20$ $(85 \%)$ of those with episodic hypoxia had Lown grade III or higher arrhythmias, whereas only $3 / 14(21 \%)$ of those without episodic hypoxia had such arrhythmias. Of those 20 patients with Lown grade III or higher arrhythmias, all but three $(85 \%)$ also had episodic hypoxia. The mean dip frequency was significantly higher in those with high grade arrhythmias than in those without such arrhythmias $(6.7 v 2.2, \mathrm{p}<0.01)$. 
A

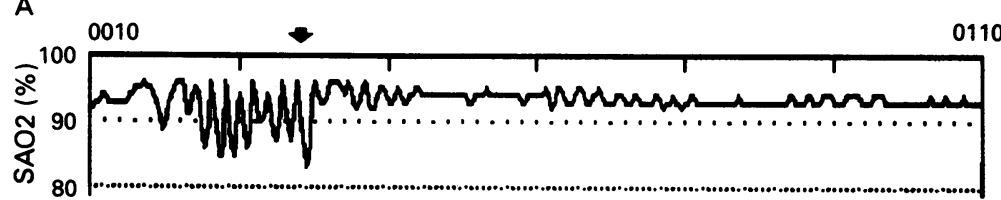

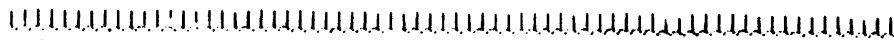

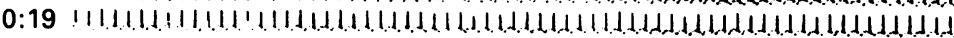

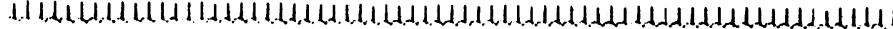

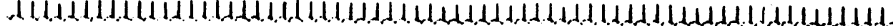

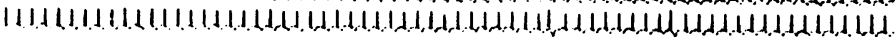

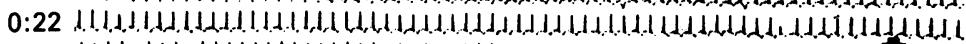

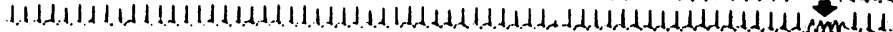

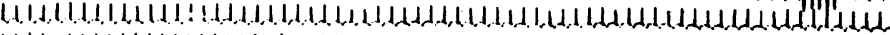



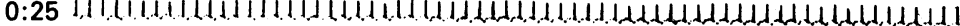

B

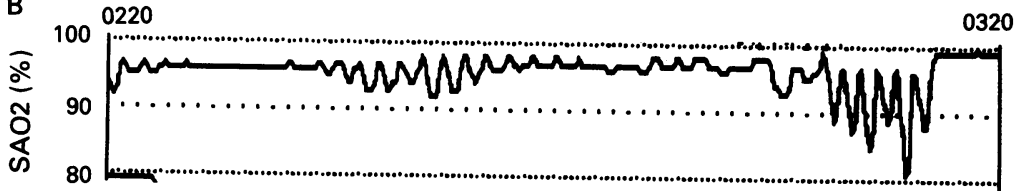

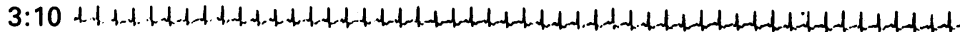

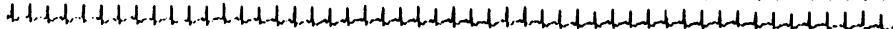

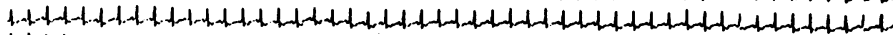

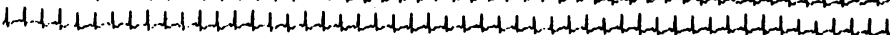

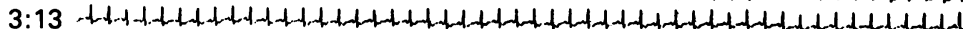

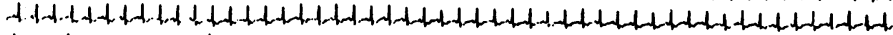

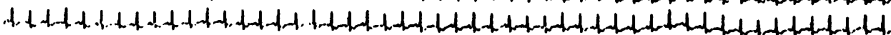

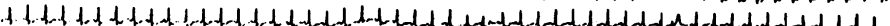

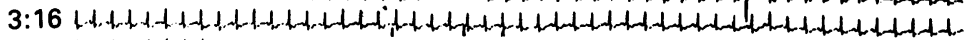

C

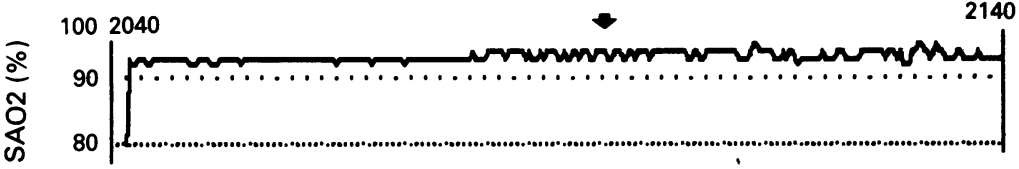

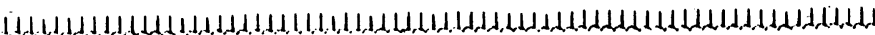

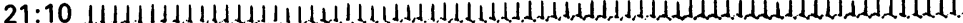

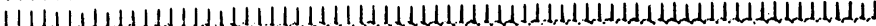

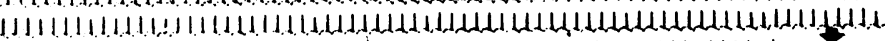

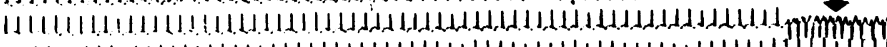

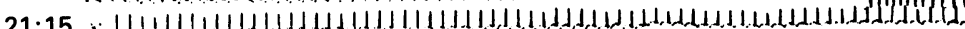

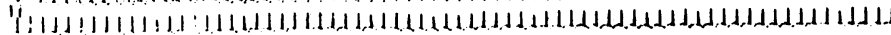

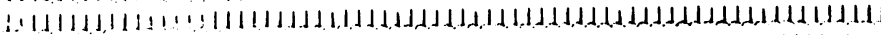

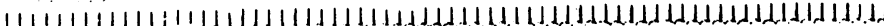

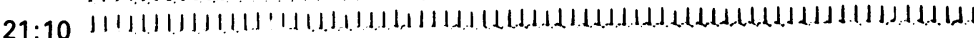

Figure 3 Inconstant temporal correlation between episodic desaturation and arrhythmias. (A) Non-sustained ventricular tachycardia (arrow) occurs between 0023 and 0024 towards the end of an 11 minute period of episodic hypoxia, $(B)$ in a differen patient, there is no arrhythmia despite considerable episodic hypoxia between 0310 and 0316 ; (C) there is non-sustained ventricular tachycardia just before 2113 (arrow) without corresponding hypoxia. Upper trace, oxygen saturation (SAO2); lower trace, pulse rate. have ejection fractions $>50 \%$ despite a clinical record suggestive of poor left ventricular function. Reversible ischaemia or diastolic dysfunction may have been predominant mechanisms in these cases. These three cases were included in the analysis despite the evidence of good systolic function although none had hypoxic episodes and their inclusion does not materially affect the results.

We have not found a causal relation between nocturnal desaturation and cardiac arrhythmias. High grade arrhythmic activity may occur during or just after a period of episodic hypoxia, but arrhythmias and desaturation episodes may be independent (fig 3). More detailed polysomnographic studies are indicated to elucidate the temporal relations between arrhythmias and episodic hypoxaemia, which probably includes an interaction with other variables, such as the prevailing sympathovagal balance, myocardial ischaemia, and other factors. Nor have we tested the effect of interventions (for example, oxygen treatment) designed to reduce hypoxia on the relations between arrhythmias and oximetric abnormalities. It has been shown that medical treatment of heart failure, ${ }^{9}$ administration of oxygen, ${ }^{10}$ and nasal continuous positive airway pressure $^{11}$ reduce nocturnal desaturation in patients with heart failure, and it might be expected that frequency of arrhythmia would be reduced in patients susceptible to arrhythmias by such measures.

None of the patients we studied had sustained, life threatening arrhythmias during the monitoring period and it is not certain that the non-sustained arrhythmias we found were of prognostic significance. Several studies suggest, however, that such arrhythmias are associated with an adverse prognosis in patients with impaired left ventricular function ${ }^{13-15}$ and it might be that correction of nocturnal desaturation would be a more effective way of preventing the development of life threatening arrhythmias than the use of potentially harmful antiarrhythmic agents. Sleep apnoea has been associated with increased mortality in unselected patients after myocardial infarction. ${ }^{16}$

In conclusion, it appears that nocturnal oxygen desaturation is common in patients with severely impaired left ventricular function and that such desaturation is associated with high grade non-sustained ventricular arrhythmias. This association does not, however, seem to be a reflection solely of severity of disease, reflected by left ventricular ejection fraction or NYHA class. Further study is required to find the mechanism of this effect and the result of interventions that limit desaturation upon arrhythmias and prognosis.

This correlation was, however, not merely a reflection of left ventricular function. The mean ejection fraction was no different between those with and without high grade arrhythmias, and there was also no significant difference between the mean dip frequency in those with grade IV NYHA dyspnoea and those with grade III dyspnoea, confirming the clinical finding that the severest cases were not exclusively associated with episodic hypoxia. It was surprising that three patients were found to
1 Trask $\mathrm{CH}$, Cree EM. Oximeter studies on patients with chronic obstructive emphysema, awake and during sleep. N Engl J Med 1962.266:639-42.

2 Phillipson EA, Goldstein RS. Breathing during sleep in patients with chronic obstructive pulmonary disease. Chest 1984;85:24-30.

3 Tilkian AG, Guilleminault, Schroeder JS, et al. Sleepinduced apnoea syndrome. Prevalence of cardiac arrhythmias and their reversal after tracheostomy. Am J Med 1977;63:348-58. 
4 Guilleminault C, Connolly SJ, Winkle RA. Cardiac arrhythmias and conduction disturbances during sleep in 400 patients with sleep apnoea syndrome. Am J Cardiol patients with

5 Holmes J, Kubo SH, Cody RJ, Kligfield P. Arrhythmias in ischaemic and nonischaemic dilated cardiomyopathy: prediction of mortality by ambulatory electrocardiography. Am J Cardiol 1985;55:146-51.

6 Spielman SR, Greenspan AM, Kay HR, et al. Electrophysiological testing in patients at high risk of sudden death. I. Nonsustained ventricular tachycardia and abnormal ventricular function. $J$ Am Coll Cardiol 1985;6:31-9.

7 Warley ARH, Stradling JR, Mitchell J. Evaluation of Ohmeda 3700 pulse oximeter. Thorax 1987;42:892-6.

8 Otsuka K, Sadakane N, Ozawa T. Arrhythmogenic properties of disordered breathing during sleep in patients with cardiovascular disorders. Clin Cardiol 1987;10:771-82.

9 Dark DS, Pingleton SK, Kerby GR, et al. Breathing pattern abnormalities and arterial oxygen desaturation during sleep in the congestive heart failure syndrome. Improvement following medical therapy. Chest 1987; 91:833-6.
10 Hanly PJ, Millar TW, Steljes DG, et al. Respiration and abnormal sleep in pa Takasaki Y, Orr D, Popkin J, et al. Effect of nasal continuous positive airway pressure on sleep apnoea in congestive heart failure. Am Rev Respir Dis 1989;140:1578-84.

12 Davies SW, John LM, Wedzicha JA, Lipkin DP. Overnight studies in severe chronic left heart failure: arrhythmia and oxygen desaturation. $\mathrm{Br}$ Heart $J$ 1991;65:77-83.

13 Huang SK, Messer JV, Denes P. Significance of ventricula tachycardia in idiopathic dilated cardiomyopathy: observations in 35 patients. Am J Cardiol 1983;51:507-12. 14 Meinertz T, Hofmann T, Kasper W, et al. Significance of ventricular arrhythmias in idiopathic dilated cardiomyopathy. Am J Cardiol 1984;53:902-7.

15 Holmes J, Kubo SH, Cody RJ, Kligfield P. Arrhythmias in ischemic and nonischemic dilated cardiomyopathy: prediction of mortality by ambulatory electrocardiography. Am J Cardiol 1985;55:146-51.

16 Hung J, Whitford EG, Parsons RW, Hillman DR. Association of sleep apnoea with myocardial infarction in men. tion of sleep apnoea with
$\underline{\underline{\underline{T}}}$

$\stackrel{\frac{1}{\mathbb{D}}}{\stackrel{1}{7}}$

c.

ç

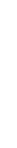

